\title{
Identification of emission lines in the low-ionization strontium filament near Eta Carinae ${ }^{\star, \star \star}$
}

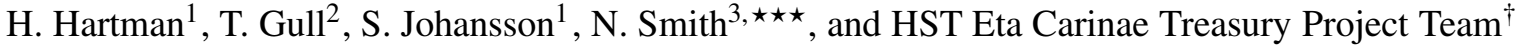 \\ 1 Atomic Astrophysics, Lund Observatory, Lund University, Box 43, 22100 Lund, Sweden \\ ${ }^{2}$ Laboratory for Astronomy and Solar Physics, Code 681, Goddard Space Flight Center, Greenbelt, MD 20771, USA \\ 3 CASA, 389 UCB, University of Colorado, Boulder, CO 80309, USA
}

Received 7 November 2003 / Accepted 10 February 2004

\begin{abstract}
We have obtained deep spectra from 1640 to $10100 \AA$ with the Space Telescope Imaging Spectrograph (STIS) of the strontium filament, a largely neutral emission nebulosity lying close to the very luminous star Eta Carinae and showing an uncommon spectrum. Over 600 emission lines, both permitted and forbidden, have been identified. The majority originates from neutral or singly-ionized iron group elements ( $\mathrm{Sc}, \mathrm{Ti}, \mathrm{V}, \mathrm{Cr}, \mathrm{Mn}, \mathrm{Fe}, \mathrm{Co}, \mathrm{Ni}$ ). $\mathrm{Sr}$ is the only neutron capture element detected. The presence of Sr II, numerous strong Ti II and V II lines and the dominance of Fe I over Fe II are notable discoveries. While emission lines of hydrogen, helium, and nitrogen are associable with other spatial structures at other velocities within the Homunculus, no emission lines from these elements correspond to the spatial structure or velocity of the Sr filament. Moreover, no identified $\mathrm{Sr}$ filament emission line requires an ionization or excitation energy above approximately $8 \mathrm{eV}$. Ionized gas extends spatially along the aperture, oriented along the polar axis of the Homunculus, and in velocity around the strontium filament. We suggest that the strontium filament is shielded from ultraviolet radiation at energies above $8 \mathrm{eV}$, but is intensely irradiated by the central star at wavelengths longward of $1500 \AA$.
\end{abstract}

Key words. line: identification - stars: circumstellar matter - stars: kinematics - stars: individual: Eta Carinae

\section{Introduction}

The Luminous Blue Variable star (LBV) Eta Carinae ( $\eta$ Car) experienced a major outburst in the 1840's with a secondary outburst in the 1890's (Davidson \& Humphreys 1997, and references therein). Several solar masses of material were ejected, which is now directly seen as the expanding Homunculus (Morse et al. 1998; Smith et al. 2003b) and the Little Homunculus (Ishibashi et al. 2003). Although the central star provides $5 \times 10^{6} L_{\odot}$ at a characteristic temperature of $25000 \mathrm{~K}$, most of the gas in the Homunculus is neutral (Davidson et al. 2001). The hollow bipolar lobes with an intervening equatorial skirt are seen primarily by dust-scattered radiation from $\eta$ Car. STIS CCD spectra, recorded with a long aperture $\left(52^{\prime \prime} \times 0\right.$. ' 2$)$,

Send offprint requests to: $\mathrm{H}$. Hartman,

e-mail: henrik.hartman@astro.lu.se

* Based on observations made with the NASA/ESA Hubble Space Telescope, obtained at the Space Telescope Science Institute, which is operated by the Association of Universities for Research in Astronomy, Inc., under NASA contract NAS5-26555.

$\star \star$ Tables 2 and 3 are only available in electronic form at the CDS via anonymous ftp to cdsarc.u-strasbg.fr $(130.79 .128 .5)$ or via http: //cdsweb.u-strasbg.fr/cgi-bin/qcat?J/A+A/419/215

$\star \star \star$ Hubble fellow.

$\dagger$ This research is partly based on data from the Eta Carinae Hubble Space Telescope Treasury project via grant No. GO-9420 from Space Telescope Science Institute. revealed a thin, interior skin in the light of [Fe II] and [Ni II], but no H II nor He I, emission. Davidson et al. (2001) used these lines, and the absorption $\mathrm{H}$ and $\mathrm{K}$ lines of $\mathrm{Ca}$ II, to trace the inner, neutral surface of the Homunculus. Ground-based observations in the near-infrared (Smith 2002) revealed molecular hydrogen in the cool exterior of the Homunculus shell. Lines of Fe II, $\mathrm{H} \alpha$ and [Ni II] revealed an internal emission nebulosity, called the Little Homunculus (Gull \& Ishibashi 2001; Ishibashi et al. 2003). Several bright, ionized emission structures exist very close to $\eta$ Car, known as the Weigelt Blobs B, C and D (Weigelt \& Ebersberger 1986), are located within 0.' 1 to 0 '!3 from the central star.

Damineli (1996) identified the 5.52-year period in the highexcitation nebular and stellar emission lines of $\eta$ Car and its surrounding nebulosity. In a long-term monitoring series of programs to understand this variability, the Weigelt blobs B and D, along with $\eta$ Car, have been observed with HST/STIS at nearly annual intervals since 1998 (Davidson et al. 1999; Gull et al. 2001). Over 2000 emission lines of the Weigelt blobs B and D were identified in the spectrum between $1700 \AA$ and $10300 \AA$ by Zethson (2001). Changes between the spectroscopic minimum in 1998 and the broad maximum during 1999 and 2000 demonstrate that lines of higher ionization disappear during the spectroscopic minimum only to reappear as the system recovers. Many H Ly $\alpha$-pumped Fe II lines appear during the maximum (Johansson \& Hamann 1993; Zethson 2001), 

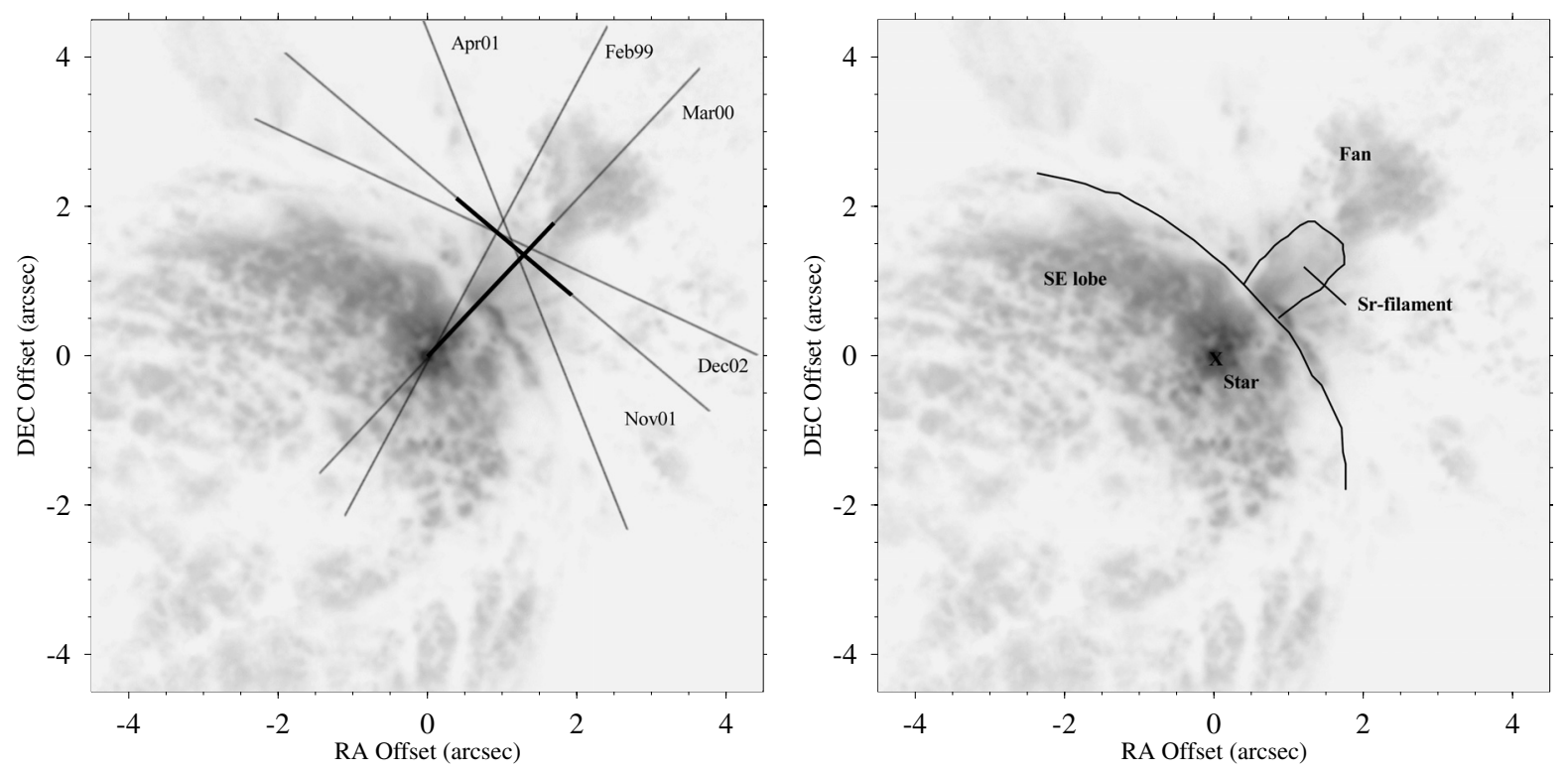

Fig. 1. ACS/HRC image of $\eta$ Car and the Homunculus (Smith et al. 2004) with the aperture positions superimposed (see Table 1). The sections plotted in Figs. 2 and 3 are marked with bold lines. The March 2000 aperture represents the aperture centered on $\eta$ Car. At this same epoch, multiple exposures were recorded with offsets orthogonal to the aperture to map the nebular structure (Ishibashi et al. 2003). We have used these same spectra to estimate the size of the Sr filament in Sr II, sketched in the right panel.

and disappear during the minimum. The Fe II 2507, $2509 \AA$ lines are the most enhanced of these fluorescence lines, and they feed long-lived Fe II states involved in a closed radiative cycle showing stimulated emission (Johansson \& Letokhov 2003). Verner et al. (2002) used the CLOUDY model to predict the optical Fe II emission fluxes of the Weigelt B and D blobs during the spectroscopic minimum event of 1998.

During a preliminary test for the Homunculus mapping program in the 6400-7000 $\AA$ region planned as a STIS GTO Key Project (HST proposal 8483), we noticed some very faint, narrow emission lines located $1.5^{\prime \prime}$ to the Northwest of $\eta$ Car. The $1^{\prime \prime}$ long emission filament appeared not to be associated with any known structure in $\eta$ Car. Yet the spatial and velocity structure was similar for these lines and indicated that they must originate from the same volume. Zethson et al. (2001) identified twenty of twenty one lines in the 6400-7000 ̊ region, all originating from a structure moving at $-100 \mathrm{~km} \mathrm{~s}^{-1}$. As the most spectacular discovery was the first identification of two [Sr II] lines, the filament became known as the $\mathrm{Sr}$ filament. Peculiarly, no lines of hydrogen or helium were identified in the spectrum of this system. While lines of Fe II were not identified, lines of Fe I were. However, from the identifications of this wavelength limited spectrum it was not possible to conclude whether these emission lines were due to a selective excitation mechanism or to different elemental abundances.

The limited spectrum of the Sr filament differed remarkably from similar spectra of other emission line nebular structures around $\eta$ Car. This fact led to additional observations and line identifications in other wavelength regions. In the present paper we report on all HST observations obtained so far of the $\mathrm{Sr}$ filament and tabulate all the measured emission lines. Nearly 600 lines have been identified, and only a few strong lines remain unidentified. We also discuss the peculiarities found in the spectrum in terms of apparent enhancements and depletions in elemental abundances, as well as clear indications of special ionization and excitation conditions in the filament.

\section{Observations}

The initial 6480 to $7000 \AA$ A spectrum of the Sr filament differed markedly from spectra of other emission line structures around $\eta$ Car, and indeed from spectra of other nebulae. [Sr II] emission is not known to have been observed in other emission nebulae. Given the uniqueness of this nebular spectrum, we followed up with a series of observations, first to detect Sr II lines near $4000 \AA$, then other nebular emission lines, within visits scheduled for $\eta$ Car. Information on these visits are listed in Table 1. The two resonance lines of Sr II at $4078 \AA$ and $4216 \AA$ were observed in emission. Bautista et al. (2002) found the Sr II line ratios to be consistent with a gas having electron densities of $10^{7} \mathrm{~cm}^{-3}$ in a predominantly neutral region. We extended the spectral coverage across the entire range of the STIS CCD (1640 to $10100 \AA$ ), and examined the spectroscopic maps of the Homunculus in spectral intervals containing $\mathrm{H} \alpha$ and $\mathrm{H} \beta$ to determine the spatial extent of the peculiar emission. The observations were done during several HST visits. As the HST spacecraft orientation changes throughout the year, we had to accept observations through the long aperture at very different position angles (Fig. 1). When possible, the aperture was centered on a common position offset 1".5 at Position Angle $315^{\circ}$ from $\eta$ Car. Enough overlap in repeat spectral coverage (Table 1) allowed us to gain significant information on the spatial extent of the $\mathrm{Sr}$ filament.

Direct imagery of the $\mathrm{Sr}$ filament is not possible through the broad-bandpass filters available in the WFPC2 or the ACS cameras. The relatively weak emission lines 
Table 1. Log of observations with the STIS.

\begin{tabular}{|c|c|c|c|c|c|}
\hline Date & $\begin{array}{l}\text { HST } \\
\text { Proposal }\end{array}$ & $\begin{array}{l}\text { Offset } \\
\text { from } \eta \text { Car } \\
R\left({ }^{\prime \prime}\right), \theta\end{array}$ & $\begin{array}{l}\text { Pos angle } \\
\text { Degrees } \\
\text { (N through } \mathrm{E})\end{array}$ & Aperture & $\begin{array}{l}\text { Spectral coverage } \\
\AA\end{array}$ \\
\hline Feb. 21, 1999 & 8036 & $0.4,45^{\circ}$ & $-27.97^{\circ}$ & $52^{\prime \prime} \times 0^{\prime \prime} 1$ & $6480-7000 \AA^{1}$ \\
\hline Mar. 13, 2000 & 8327 & $0,0^{2}$ & $-41.14^{\circ}$ & $52^{\prime \prime} \times 00^{\prime} 2 \mathrm{~F} 2$ & $\begin{array}{l}2480-2910 \AA \\
3795-4335 \AA \\
4818-5100 \AA \\
6480-7565 \AA \\
9300-9600 \AA\end{array}$ \\
\hline Mar. 21, 2000 & 8483 & $\begin{array}{l}N \times 0.1,55^{\circ 5} \\
N \times 0.25,55^{\circ 5}\end{array}$ & $-35.02^{\circ}$ & $52^{\prime \prime} \times 0 \prime^{\prime} 1$ & $\begin{array}{l}4818-5100 \AA \\
6480-7050 \AA\end{array}$ \\
\hline Apr. 17, 2001 & 8619 & $1^{\prime \prime} 5,315^{\circ}$ & $22.06^{\circ}$ & $52^{\prime \prime} \times 0.2$ & $\begin{array}{l}4052-4593 \AA^{6} \\
4818-5100 \AA \\
6480-7050 \AA^{3}\end{array}$ \\
\hline Nov. 27, 2001 & 8619 & $1^{\prime \prime} 5,315^{\circ}$ & $-130.97^{\circ}$ & $52^{\prime \prime} \times 0 \prime^{\prime} 2$ & $\begin{array}{l}2480-2633 \AA \\
3022-10135 \AA^{3,4,6}\end{array}$ \\
\hline Dec. 16, 2002 & 9420 & $1^{\prime \prime} .5,315^{\circ}$ & $-114.94^{\circ}$ & $52^{\prime \prime} \times 0.2$ & $\begin{array}{l}1640-2492 \AA \\
2897-3052 \AA\end{array}$ \\
\hline
\end{tabular}

${ }^{1}$ Initial discovery spectrum (Zethson et al. 2001).

${ }^{2}$ Mar. 13, 2000 observations were accomplished with the F2 (0'.85) fiducial blocking the $\eta$ Car at the STIS entrance.

${ }^{3}$ Repeated spectra from $6480-7000 \AA$ to check for variability.

${ }^{4}$ Repeated spectra at $6995-7565 \AA$ to check variability.

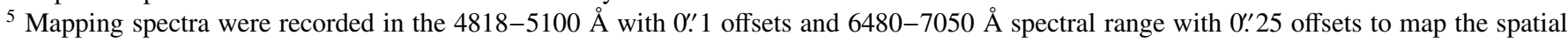
structure of the Homunculus and the Little Homunculus. We used these spectra to estimate the size of the strontium filament in Fe II and [Sr II]. ${ }^{6}$ Repeated spectra 4194-4593 Å; 4818-5104 Å to check variability.

are overwhelmed by dust-scattered starlight throughout the Homunculus. Other nebular emission structures with different photo-excitations, different spatial and velocity intervals are located in, or close to, the line of sight towards the $\mathrm{Sr}$ filament. With direct imagery as in Fig. 1 (Morse et al. 1998; Smith et al. 2004), we can trace the dusty structures of the Homunculus by the scattered, red starlight. Polarization measures using WFPC2 imagery (Schulte-Ladbeck et al. 1999; King et al. 2002) confirm the scattering properties of this light. The Little Homunculus was detected by multiple emission lines of Fe II, Cr II, etc. in the near-ultraviolet, and may contribute to the "purple haze", associated with the feature commonly called the "Fan" within the Northwest lobe (Smith et al. 2004). Within the skirt, or the gas and dust structure located between the two lobes, bright emission lines extend over significant regions. Inspection of these emission lines indicate that some emission extends, in velocity and space, around the Sr filament (Fig. 2). The $\mathrm{Sr}$ filament is best mapped with high spatial and moderate spectral resolution as produced by the STIS CCD moderate dispersion modes. Ground-based observations are currently limited to half-arcsecond seeing with much scattered light from the central star. The Sr filament structure then becomes confused with other nebular emission and stellar emission.

Spectra of the Sr filament were recorded through HST visits scheduled between February 1999 to December 2002 (2.8 years). This extends over the mid-portion of $\eta$ Car's broad spectroscopic maximum, which covers 5 years of the 5.52 year period. Highly excited emission lines of [Ne III], [Ar III] and He I, as monitored of the entire nebulosity by
Damineli et al. (1998), changed slowly over this interval in nebular structure close to $\eta$ Car (but these lines are not detected in the Sr filament).

Given that these observations occur late in the broad spectroscopic maximum (when fluxes of all nebular lines appear to be relatively constant), we do not anticipate significant changes in the excitation of the Sr-filament. The spectrum of the Weigelt Blobs, being at an order of magnitude closer to the Central Source, shows little change in the low-excitation emission lines of e.g. Fe II and Ni II across the entire 5.52-year cycle. Modeling by Verner et al (2002 and in preparation) demonstrates that the low-excitation emission lines are due largely to UV radiation longward of Lyman alpha. Most excitation of the Sr filament appears to be due to mid-UV and near-UV, which changes little across the minimum. Smith et al. (2000) investigated the photometric variability in the "purple haze" in WFPC2 pictures and found no evidence for variability that one might associate with the Sr filament, even during the 5.5 year cycle and the brightening of the star.

Where possible we repeated some spectral overlap to check for variability in emission line fluxes. The specific dates, HST programs, offsets from $\eta$ Car, position angle, STIS aperture and spectral coverage are listed in Table 1. Locations of the aperture positions are overlaid on an Advanced Camera for Surveys (ACS) High Resolution Camera (HRC) ultraviolet image of the Homunculus (Smith et al. 2004) in Fig. 1.

The discovery spectrum was recorded on February 21, 1999 under program 8036 (Zethson et al. 2001). Under program 8327, deep spectra were recorded on March 13, 2000 

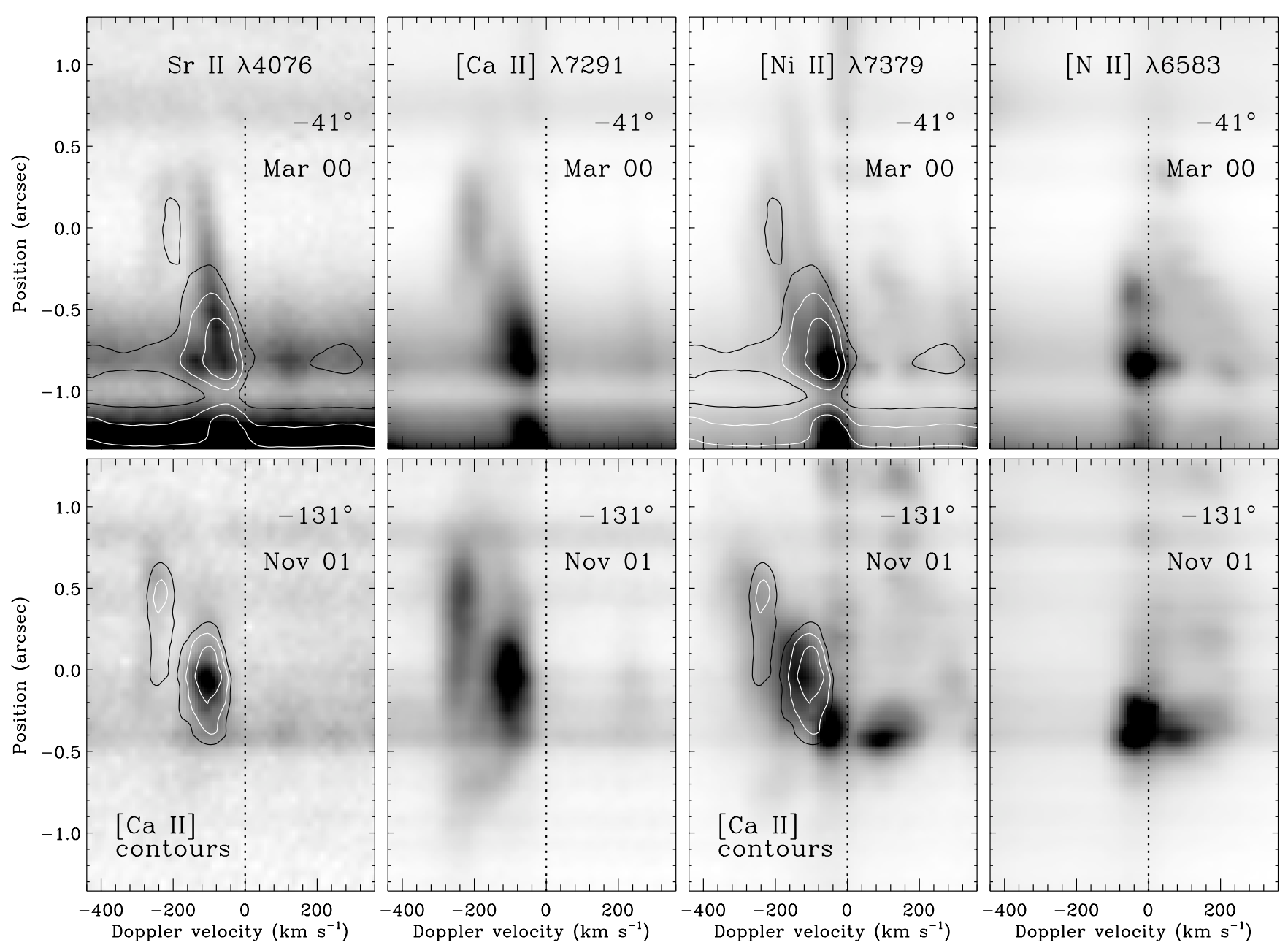

Fig. 2. Position-velocity diagrams at the location of the strontium filament seen in several different emission lines, with two different slit orientations. The top row shows spectra taken in March 2000 with the STIS slit at PA $=-41^{\circ}$, and the bottom row shows the same four emission lines obtained in November 2001, with the slit at PA $=-131^{\circ}$ (see Fig. 1 and Table 1). The four emission lines shown here are (left to right in both the top and bottom panels): Sr II $\lambda 4078$, [Ca II] $\lambda 7293$ (this is actually the average of [Ca II] $\lambda 7293$ and [Ca II] $\lambda 7325$ ), [Ni II] $\lambda 7379$, and [N II] $\lambda 6585$. The contours superposed on the Sr II and [Ni II] emission are corresponding contours of the [Ca II] emission at the same dates and slit positions. The horizontal axis shows heliocentric Doppler velocity. In the top and bottom panels, the position marked as zero corresponds roughly to the place where the two slits cross to within about 0. ' 1 (see Fig. 1).

for two purposes: 1) we wanted deeper exposures of the Homunculus to infer the spatial structure of the lobes by the changes in velocity with position of locally-emitted narrow nebular emission lines and the locally-absorbed narrow absorption lines of Ca II (Davidson et al. 2001), and 2) we wanted to extend the spectral coverage of the $\mathrm{Sr}$ filament to other spectral regions as we anticipated that emission lines from additional elements in neutral and singly-ionized states would be detected. As most emission lines in the Homunculus are marginally resolved with the CCD G750M, G430M and G230MB gratings and the $52^{\prime \prime} \times 0$.' 1 aperture, a significant gain in limiting flux was obtained by using the $52^{\prime \prime} \times 0.2$ aperture. For this visit with STIS, we placed $\eta$ Car behind the F2 fiducial ( 0. ' 85 wide), to prevent saturation of the CCD by the very bright star. On March 21, 2000 (program 8483), the Homunculus was mapped with the STIS using the $52^{\prime \prime} \times 0$.' 1 aperture and the grating settings for 6480-7000 $\AA$ at 0.25 spacing and for 4818-5100 at 0.1 spacing. The overall structure, with emphasis on the internal ionized regions called the Little Homunculus, is discussed by Ishibashi et al. (2003). We used these same data to map the Sr filament. Deep spectra were recorded in April 2001 (program 8619) at wavelengths selected to obtain the fluxes of Sr II emission lines near $4100 \AA$, to measure the width of the $\mathrm{Sr}$ filament, and to detect additional emission lines. On November 27, 2001 (Program 8619), we recorded deep exposures from $3022-10135 \AA$ and $2480-2633 \AA$ as that was the region of the spectrum where many emission lines were predicted, including the Fe II lines at $2507 \AA$ and $2509 \AA$. Based upon the non-detection of the Lyman-alpha-pumped Fe II lines at 2507 and $2509 \AA$, we suspected that little or no hydrogen photo-ionizing radiation was impinging upon the $\mathrm{Sr}$ filament. We expected that few emission lines would be detected below $2480 \AA$ A. On December 16, 2002, we extended spectral coverage from $3052 \AA$ to $1640 \AA$ to gain full spectral coverage. No lines were detected shortward of $2489.7 \AA$, which is an Fe I line of multiplet uv9. However, we caution the reader that 
the CCD sensitivity decreases rapidly below $2500 \AA$ A. Deeper exposures would be possible with the MAMA detectors, likely in the E230M mode.

\section{Kinematic structure of the strontium filament}

The structure of the strontium filament is complex, and varies depending on the particular emission line observed. Since several distinct emission-line structures are seen projected along the same line of sight, the morphology needs to be understood before we can attempt to interpret the observed spectrum and assign line identifications. We know from previous study (Davidson et al. 2001; Smith 2002; Ishibashi et al. 2003) that at the position of the strontium filament there are at least three different structures: the equatorial skirt, the receding northwest polar lobe of the Homunculus (showing both intrinsic emission and reflected light), and the Little Homunculus. Fortunately, these different features can be disentangled by considering their Doppler shifts.

Figure 2 shows the kinematic structure at the position of the strontium filament. Two different position angles, perpendicular to one another, are chosen to sample its full spatial extent (see Fig. 1). The resonance line Sr II $\lambda 4078$ is the brightest of the four lines from $\mathrm{Sr}^{+}$that have been detected in our data (Zethson et al. 2001). [Ca II] is a much brighter line that traces gas in a similar ionization state; $\mathrm{Ca}^{+}$ranges from 6.1 to $11.9 \mathrm{eV}$, while $\mathrm{Sr}^{+}$has a range of 5.7 to $11.0 \mathrm{eV}$. Indeed, contours of [Ca II] superposed over Sr II emission show that both lines seem to trace the same gas ${ }^{1}$. The [Ca II] emission shows that the Sr filament is more than just a single thin "filament", and has a spatial extent of $\gtrsim 11^{\prime \prime} 5$ along the polar axis of the Homunculus, and $\gtrsim 2^{\prime \prime}$ in the direction perpendicular to the polar axis. Thus, the $\mathrm{Sr}$ filament occupies the same spatial extent as the "purple haze" seen in HST images of $\eta$ Car (Smith et al. 2004; Morse et al. 1998). The Sr filament is constrained to heliocentric velocities between -50 and $-300 \mathrm{~km} \mathrm{~s}^{-1}$, and is probably in or near the equatorial plane. It appears to have two main velocity components; one at about $-100 \mathrm{~km} \mathrm{~s}^{-1}$, with a velocity structure tilted so that emission becomes more blueshifted with increasing separation from the star (in March 2000), and another feature at about $-240 \mathrm{~km} \mathrm{~s}^{-1}$ with the opposite tilt. The $-100 \mathrm{~km} \mathrm{~s}^{-1}$ component is brighter at most positions (at least in $\mathrm{Sr}$ II), and dominates the emission spectrum listed in Table 2. Interestingly, fainter extended emission in [Ca II] suggests that both these velocity components may be part of a single closed structure, forming a ring or loop in velocity space, especially in the November 2001 spectra.

Emission from [Ni II] $\lambda 7379$, on the other hand, shows subtle differences compared to both $\mathrm{Sr}$ II and [Ca II]. It has two remarkably straight velocity components (top panel in Fig. 2), both tilted in the same sense, both with blueshift increasing with separation from the star. Davidson et al. (2001) suggested that these two velocity components traced gas in the equatorial plane with two different ages, originating in the Great Eruption

\footnotetext{
${ }^{1}$ In regions exposed to radiation above 11 to $12 \mathrm{eV}, \mathrm{Sr}$ and $\mathrm{Ca}$ will both be doubly ionized and difficult to detect because of their atomic structure.
}

and the 1890 event (see Humphreys et al. 1999). Some of the [Ni II] emission coincides with Sr II and [Ca II], but some does not. In particular, the diffuse [Ni II] emission near position $=0^{\prime \prime}$ and $-150 \mathrm{~km} \mathrm{~s}^{-1}$ in the bottom panel of Fig. 2 seems to fill in the gap between the two velocity components of Sr II and [Ca II]. Perhaps this makes sense, since $\mathrm{Ni}^{+}$occupies ionization zones between 7.6 and $18.2 \mathrm{eV}$, only partly overlapping with $\mathrm{Sr}^{+}$and $\mathrm{Ca}^{+}$. The velocity components of [Ni II] and [N II] at $-40 \mathrm{~km} \mathrm{~s}^{-1}$ and redshifted velocities up to $-300 \mathrm{~km} \mathrm{~s}^{-1}$ trace the northwest polar lobes of the Homunculus and Little Homunculus, respectively (Ishibashi et al. 2003; Smith et al. 2004). [N II] emission is only seen in these polar features, and is absent in the equatorial material, while [Ni II] is seen in both. These polar structures must be exposed to radiation above $12 \mathrm{eV}$, since Sr II and [Ca II] are absent. This reinforces the idea that the strontium filament is somehow shielded from radiation above $12 \mathrm{eV}$ (even though the ionization potential of $\mathrm{N}$ is $14.5 \mathrm{eV}$, it can be ionized from the excited ${ }^{2} \mathrm{D}$ state by photons at $\sim 12.1 \mathrm{eV}$ ).

As noted above, the blueshifted velocities imply that the strontium filament may reside in or near the equatorial plane this may be an important factor for understanding its unusual excitation. On the one hand, various clues suggest that $\eta \mathrm{Car}$ has an asymmetric radiation field, with more UV radiation escaping the stellar wind at low latitudes near the equator where the wind is thinner (Smith et al. 2003a, 2004), at least during its "normal" high-excitation state between spectroscopic events. On the other hand, there may be a considerable column of material between the star and the strontium filament, including the Weigelt objects and a larger-scale disk or torus, which may absorb all ionizing photons along that path but apparently transmits photons below $12 \mathrm{eV}$. In any case, both the strontium filament and the Weigelt objects appear to occupy a special azimuthal direction relative to $\eta$ Car. In general, the subtle variations in emission structure from one tracer to the next suggest that the strontium filament is a low-ionization region with stratified ionization zones. This will be relevant in future efforts to model the emission spectrum (Bautista et al., in preparation).

During the April 2001 observation, the slit was oriented in a way that a region $\mathrm{NE}$ of the filament (i.e. $\sim 2$ " north of the star) was observed. This spatial region close to the filament shows a weak scattered continuum. In this continuum can be seen absorption lines from the allowed Sr II lines $\lambda 4078,4216$ and the $\mathrm{Ca}$ I $\lambda 4227$ line. For this slit orientation, there are only a few wavelength regions observed, but this absorption is not observed in any other lines. For all of the transitions showing this absorption, the lower level is the ground state, which might indicate that this is a low excitation region.

\section{Line identification}

The spectra of the Sr filament were observed at five different dates with a long aperture at different position angles as mentioned in Sect. 2 (see Fig. 1 and Table 1). By inspection of individual visits, we know that there are very significant spatial variations of the nebular emission. Given the interval in time between all observations, there is the 

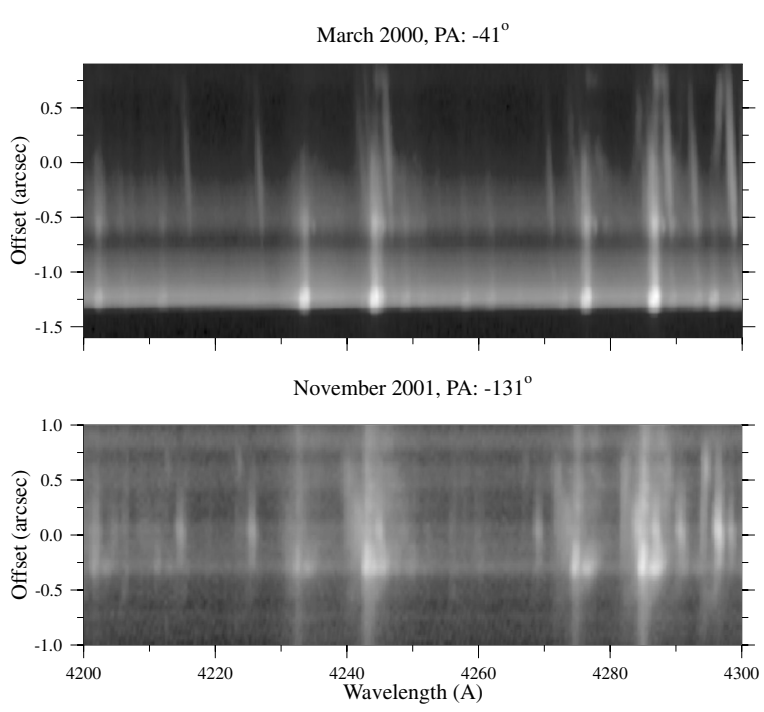

Fig. 3. 2D spectrum of the filament at two different slit orientations at nearly orthogonal angles, March 2000 and November 2001, respectively. The vertical stretch in the upper panel is $22^{\prime \prime} 5$ and in the lower $2^{\prime \prime}$. The horizontal scale covers the region 4200-4300 ̊. The aperture width was 0.2 on the sky. The slit positions are shown projected on the Homunculus in Fig. 1 with the plotted regions marked with bold lines.

possibility of temporal variations. Other than in the region of close overlap, there is also the strong possibility of spatial and/or temporal variations complicating the application of line ratios, especially if the relevant spectral lines were not measured in the same visit. However, some spectral regions were observed on several occasions. The different profiles for the same spectral line in different visits can then be compared. We also note that the bulk of the observations were taken thirteen months apart (in November 2001 and December 2002) at position angles within $16^{\circ}$ of each other. The star is in the broad, high-excitation phase; temporal variability should be minimum across this time-span. Spatial variations appear to be small in the central core of the Sr filament.

Some observed emission lines are likely the composite of emission from a number of regions along line of sight. The imaging properties of STIS along the aperture and the radial velocity shifts in the spectral direction enable us to disentangle the nebular emission for several kinematically different systems, as discussed in Sect. 3 above and seen in Fig. 2. Lines belonging to the filament show a Doppler shift of about $-100 \mathrm{~km} \mathrm{~s}^{-1}$ at the center of the filament.

Examples of the spatial and velocity variations for two different slit orientations are presented in Fig. 3. The aperture position angle was at $-41^{\circ}$ (North through East convention) in March 2000 (upper plot) and is located in the general direction from $\eta$ Car along the polar axis of the Homunculus. In the 2 '. 5 slice (top, Fig. 3), the Sr filament is centered at $0^{\prime \prime}$. A velocity shift is noticeable in the spectral dispersion to the blue at 0.5 . The November 2001 observations was with the aperture positioned at $-131^{\circ}$, or perpendicular to the Homunculus polar axis. The peak of the $\mathrm{Sr}$ filament emission is positioned at $0^{\prime \prime}$. There is little velocity shift along the slice of nebular in this direction. In Fig. 4, a second slice of spectrum is shown for the

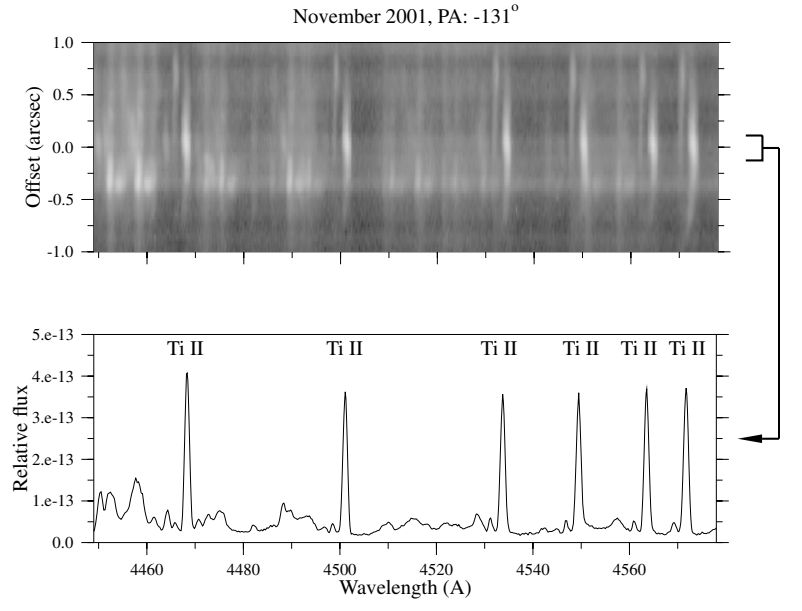

Fig. 4. Upper panel: subsection of STIS nebular spectrum centered upon the Sr filament. The STIS $52 \times 0$ '. 2 aperture was used for this spectrum. Lower panel: extracted spectrum of the $0.2 \times 0.2$ area, marked on the right hand side of the nebular spectrum in the upper panel. This represents the central position of the Sr filament.

November 2001 set of spectra (top). The extracted spectrum for a 0 .'2-high extraction is plotted below. Note that all six bright lines are Ti II emission lines of the $-100 \mathrm{~km} \mathrm{~s}^{-1} \mathrm{Sr}$ filament. A much fainter second filament can be seen to the blue in both the spectral image (top) and the extracted spectrum (bottom). These two structures are plotted in velocity in Fig. 2.

\subsection{Linelist and intensities}

All lines attributed to the $\mathrm{Sr}$ filament are presented in Table 2. The major selection criteria are the shape of the line and its spatial location in the long-slit STIS spectrum (see Sect. 3). We do not observe any lines attributable to the $\mathrm{Sr}$ filament in the region below $2480 \AA$ A. Little emission is observed below $2000 \AA$. Strong absorption along the line of sight from singly-ionized iron-group elements removes much light at these wavelengths, and the CCD sensitivity also declines in the mid-UV region. The absence of lines could also be linked to the possible presence of a lower wavelength limit for radiation available for ionization and excitation. Some faint emission lines are present but they may not be associable with the Sr filament as emission lines are present from other regions along line of sight. The region $2500-3000 \AA$ is less affected by absorption and shows emission lines. The region longward of $3000 \AA$ contains well-defined Sr filament emission lines, although emission from other spatial regions affects the spectrum.

The observed lines in Table 2 were measured in vacuo with heliocentric velocity corrections (Col. 1) and, where identified, include laboratory wavelengths (Col. 3). The difference between the two is converted to a velocity $\left(\mathrm{km} \mathrm{s}^{-1}\right.$, Col. 2). The velocities are derived from the nov01 spectrum except for a few cases, when the mar00 (2630-3025 $\AA$ ) spectra are used. The spectra include emission lines from other nebular structures in line of sight, but only the lines associated with the strontium filament are included in the linelist. Some lines have measured velocities deviating from $-100 \mathrm{~km} \mathrm{~s}^{-1}$ as they are either affected by absorption or blended by other lines. 
Blended lines, or lines with possible multiple identifications, are listed more than once with the identified wavelength, but with alternate wavelengths corresponding to the alternate line identifications. These lines are associated with the strontium filament despite the deviant Doppler velocity. The lines are identified by the species (e.g. Fe I), multiplet number and transition in Cols. 4-6. The transition is represented by the lower and upper level, using the $L S$ term notation in Moore's tables of multiplets and energy levels. Thus, the term notation is preceded by a small letter a, b, c, etc. for even parity configurations and $\mathrm{z}, \mathrm{y}, \mathrm{x}$, etc. for odd-parity configurations. If the multiplet is missing in Moore's tables we have inserted an abbreviated configuration notation. For full spectroscopic notations the reader is referred to the original laboratory line lists or to detailed atomic databases ${ }^{2}$. Intensities from the different STIS spectra are given in Cols. 7-10. Unidentified lines are marked with "unid" in the fourth column.

In Table 3 we have sorted the identified lines from Table 2 according to element, starting with the lightest element, carbon, and ending with the heaviest, strontium. Within each species the lines have been grouped after the excitation potential of the upper level, from which the line originates. The velocity for each line has been included to facilitate the study of consistency within each group of lines for a specific species.

\subsection{Observed lines}

The spectrum of the Sr filament is dominated by permitted and forbidden lines of the iron group elements, as can be seen in Table 3. The spectrum is quite different from spectra of the Weigelt blobs (Zethson 2001) as regards the line and intensity distributions among the different elements. For example, lines of Fe I, V II and Ti II are particularly strong in the Sr filament, whereas only a few Fe II lines are observed. Among the observed spectra are C I, Mg I, Al II, Ca I, Ca II, Sc II, Cr II, Mn II, Fe II, Co II, Ni II and Sr II. By contrast, lines of Fe II are dominant in the Weigelt blobs. Fe III and Fe IV lines are identified, but very few Fe I lines (Zethson 2001).

The spectral distribution of lines from a specific atom (ion) is determined by the atomic structure and the value of the ionization potential. Hence, the number of lines identified for different species reflects not only the abundance but also the complexity of the atomic structure. Assuming an upper limit of about $8 \mathrm{eV}$ of the photon energy available for ionization and excitation (see Sect. 2) the number of observable emission lines from some spectra will be very small. We can divide the spectra of the observed species in three groups, corresponding to their atomic structure and referring to the periodic table:
I) Group $1 \mathrm{~A}$ and $2 \mathrm{~A}$ ( $\mathrm{Na} \mathrm{I,} \mathrm{Mg} \mathrm{I,} \mathrm{Al} \mathrm{II,} \mathrm{Ca} \mathrm{I,} \mathrm{Ca} \mathrm{II,} \mathrm{Sr} \mathrm{II).}$
II) Group 3A-7A (C I).
III) 3d-elements ( $\mathrm{Sc}-\mathrm{Ni})$.

Group I has quite simple spectra and the resonance lines appear in the optical region for the alkali atoms. In alkali

\footnotetext{
${ }^{2}$ E.g.http://physics.nist.gov/cgi-bin/AtData/main_asd at NIST or the data by Kurucz at http://cfa-www.harvard.edu/ amdata/ampdata/kurucz23/sekur.html
}

spectra there are no forbidden spectral lines to observe, but the alkaline earth-like ions $\mathrm{Ca}$ I and $\mathrm{Sr}$ II show forbidden $3 \mathrm{~d}-4 \mathrm{~s}$ $(4 d-5 s)$ transitions due to the similar binding energy of $d-$ and s-electrons in transition group elements. In practice, these elements could therefore be placed in group III, where the overlap of $3 \mathrm{~d}-$ and $4 \mathrm{~s}$ configurations is the characteristic signature. In general, the number of lines increases with the number of valence electrons, i.e. the atomic number, for the transition elements, but decreases towards the end of the period when the d-shell gets closed. We see in Table 3 that there is a large number of lines of Sc II, Ti II and V II compared to Cr II, Mn II and Fe II. This excess of lines for the first elements of the transition group could thus be an abundance effect. However, we have to keep in mind that the ionization energy, and therewith also the excitation energy for the resonance lines increase with atomic number for the iron-group. A limited photon energy for radiative excitation favors the lightest iron-group elements, and the wavelengths of the resonance lines is below $2480 \AA$ for the heavier ones.

Some single line identifications in Table 2 (and 3 ) are questionable either because the associated velocity differs remarkably from other lines or because the excitation energy is much higher than for other lines of the same species. However, the presence of the element is clear. As discussed above the presence of elements having a simple atomic structure is difficult to verify from the number of spectral lines. For example, aluminum is detected only by the inter-combination line of Al II and cannot be verified by other lines in the observed wavelength region, assuming similar excitation energies as for other elements. For comparison, we show in Fig. 5 the situation for two spectra, Ti II and Sr II, where all allowed transitions within the observed wavelength range are included. The Einstein $A$-value (times the statistical weight of the upper level) is given on the horizontal axis as a measure of the line strength and the excitation energy of the upper level of the transition on the vertical axis. Among all possible transitions (marked with grey dots) the observed lines are marked with black dots. An expected trend of decreasing level population with increasing excitation energy can be seen. In addition, we also clearly see the larger number of predicted lines for Ti II due to a more complex atomic structure (Ti II has three and Sr II one electron outside closed shells).

No lines from hydrogen or helium that can be associated with emission from the $\mathrm{Sr}$ filament have been detected. Neither have lines from nitrogen or oxygen. However, the spectrum includes the dust-scattered hydrogen Balmer P-Cygni stellar emission and could mask very weak nebular Balmer emission lines. The only neutron-capture element identified is strontium, but two of the unidentified lines, discussed in the next subsection, coincide in wavelength with Y II. However, they cannot be confirmed by other Y II lines having about the same probability to occur. Two of the unidentified lines coincide with the two strongest lines of the resonance multiplet of $\mathrm{Zr} \mathrm{II}, \mathrm{a}^{4} \mathrm{~F}-\mathrm{z}^{4} \mathrm{G}$. Other than a coincidence with one other weak line, no other lines from $\mathrm{Zr}$ II are observed.

The line intensities from individual observations are included in Table 2. They are represented by the integrated flux in the emission feature, where contributions from obvious 
Ti II

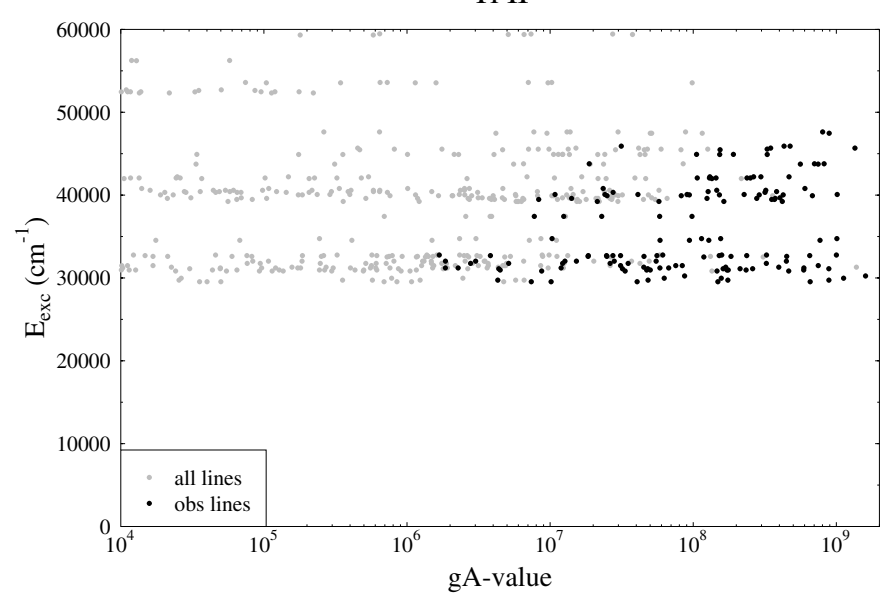

Sr II

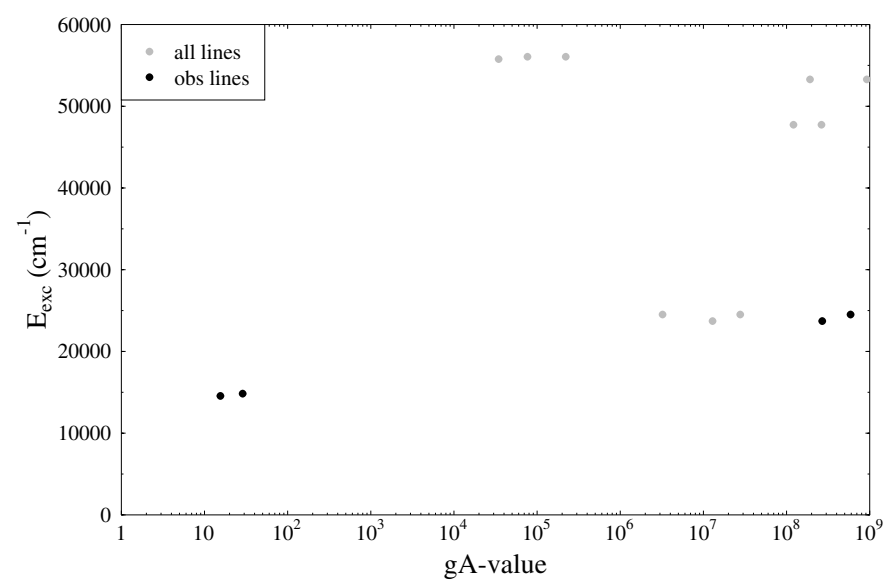

Fig. 5. Lines from Ti II (upper panel) and Sr II (lower panel) in the region 3000-10000 A for Ti II and 3000-11 $000 \AA$ for Sr II. All possible lines are shown in grey and the lines observed in the spectrum are in black. The difference in atomic structure is obvious. Note the different $x$-scales. The forbidden lines of [Ti II] are not included.

blending components have been subtracted. The tabulated flux is measured from intensity-calibrated spectra, which have not been corrected for interstellar reddening. Since the different observations do not cover the same spatial region the intensity ratios for different line pairs may not be the same in the different observations. In the wavelength regions affected by foreground absorption the intensity values are less reliable. Generally, the accuracy of the intensities is also affected by blending from other lines as well as from emission from the same line formed in other spatial regions along the line of sight. Some lines of e.g. [Fe II] and [Ni II] show complex profiles. The intensity contribution from the $\mathrm{Sr}$ filament is difficult to determine. In such cases we used multi-Gaussian fits to extract the fluxes.

\subsection{Unidentified lines}

About 40 of the 600 observed lines in the spectra of the $\mathrm{Sr}$ filament remain unidentified. These are marked with unid in Table 2 and listed in Table 4. A dozen unidentified lines have substantial strengths, as indicated in the intensity column of Table 4. For some of the lines we include possible
Table 4. Unidentified lines.

\begin{tabular}{|c|c|c|}
\hline$\lambda_{\text {obs }}(\AA)$ & Int $(\text { nov01) })^{a}$ & Wavelength coincidence \\
\hline 2537.92 & 14 & $\overline{\mathrm{bl}}$ \\
\hline 2538.90 & 22 & \\
\hline 2548.76 & 7 & VII a ${ }^{3} \mathrm{P}_{1}-\mathrm{y}^{3} \mathrm{P}_{1}$ \\
\hline 2580.24 & 37 & \\
\hline 2592.96 & 33 & bl abs \\
\hline 2815.20 & $3^{b}$ & $\mathrm{FeI} \mathrm{a}{ }^{3} \mathrm{~F}_{2}-\mathrm{sp}^{5} \mathrm{H}_{3}$ \\
\hline 3050.49 & 8 & VII a $\mathrm{a}_{3}-\mathrm{y}^{3} \mathrm{P}_{2}$ \\
\hline 3146.64 & 5 & \\
\hline 3208.63 & 8 & \\
\hline 3379.87 & 19 & TilI b ${ }^{4} \mathrm{P}_{5 / 2}-\mathrm{y}^{2} \mathrm{D}_{3 / 2}$ \\
\hline 3391.76 & 15 & $\mathrm{ZrII} \mathrm{a}^{4} \mathrm{~F}_{9 / 2}-\mathrm{Z}^{4} \mathrm{G}_{11 / 2}$ \\
\hline 3414.65 & 7 & $\mathrm{VII}^{3} \mathrm{D}_{3}-\mathrm{z}^{1} \mathrm{D}_{2}$ \\
\hline 3416.15 & 13 & \\
\hline 3438.51 & 34 & $\mathrm{ZrII} \mathrm{a}{ }^{4} \mathrm{~F}_{7 / 2}-\mathrm{Z}^{4} \mathrm{G}_{9 / 2}$, bl. \\
\hline 3600.62 & 5 & $\mathrm{YII} \mathrm{a}^{3} \mathrm{D}_{3}-\mathrm{z}^{3} \mathrm{D}_{3}$ \\
\hline 3687.65 & 11 & \\
\hline 3697.79 & 4 & \\
\hline 3709.35 & 9 & YII a ${ }^{3} D_{3}-z^{3} F_{3}$, bl. \\
\hline 3774.24 & 9 & $\begin{array}{l}\text { TiII a }{ }^{2} F_{5 / 2}-z^{4} F_{7 / 2} \\
\text { YII a }{ }^{3} D_{2}-z^{3} F_{3} \\
\text { SrII } 5 d^{2} D_{5 / 2}-7 f^{2} F\end{array}$ \\
\hline 3856.35 & 4 & \\
\hline 3958.07 & 1 & $\mathrm{ZrII} \mathrm{a}^{2} \mathrm{D}_{3 / 2}-\mathrm{Z}^{2} \mathrm{~F}_{5 / 2}$ \\
\hline 4305.24 & 6 & SrII $5 p^{2} P_{3 / 2}-6 s^{2} S_{1 / 2}$ \\
\hline 4427.25 & 2 & TiII b ${ }^{4} \mathrm{P}_{5 / 2}-\mathrm{z}^{4} \mathrm{D}_{3 / 2}$ \\
\hline 4747.17 & 9 & SrII $6{ }^{2} \mathrm{~S}_{1 / 2}-7 \mathrm{p}{ }^{2} \mathrm{P}_{1 / 2}$ \\
\hline 4763.86 & 4 & TiII a ${ }^{2} \mathrm{P}_{3 / 2}-\mathrm{z}^{4} \mathrm{~F}_{5 / 2}$ \\
\hline 4802.32 & 7 & \\
\hline 5253.20 & 1 & FeI $z^{5} D_{1}-e^{5} D_{1}$ \\
\hline 5303.74 & 3 & {$[\mathrm{FeI}] \mathrm{a}^{5} \mathrm{D}_{2}-\mathrm{a}^{3} \mathrm{P}_{1}$} \\
\hline 5470.63 & 7 & \\
\hline 5559.85 & 4 & \\
\hline 5599.35 & 1 & \\
\hline 6160.44 & 2 & \\
\hline 6219.08 & 2 & FeI a ${ }^{5} P_{2}-y^{5} D_{2}$ \\
\hline 6998.68 & 2 & \\
\hline 7426.27 & 7 & \\
\hline 7430.71 & 4 & \\
\hline 7664.71 & 13 & YII $a^{3} D-a^{3} P$ \\
\hline 7749.18 & 4 & \\
\hline 7808.06 & 6 & \\
\hline 8050.43 & 3 & \\
\hline 8674.12 & 2 & {$[\mathrm{VII}] \mathrm{a}^{3} \mathrm{~F}_{4}-\mathrm{b}^{3} \mathrm{D}_{3}$} \\
\hline 8698.34 & 2 & {$[\mathrm{VII}] \mathrm{a}^{5} \mathrm{~F}_{5}-\mathrm{a}^{3} \mathrm{G}_{5}$} \\
\hline 8806.31 & 4 & \\
\hline 9341.82 & 4 & \\
\hline
\end{tabular}

${ }^{a} 10^{-15} \mathrm{erg} \mathrm{cm}^{-2} \mathrm{~s}^{-1} \operatorname{arcsec}^{-2}$.

${ }^{b}$ The $2815 \AA$ line is not covered in the Nov01 observations. The observed intensity is from the Mar00 data.

identifications, but they should only be regarded as wavelength coincidences between observed lines and predicted transitions. The reasons for not including them among the identified lines could be a large Doppler velocity, an anomalous excitation energy or a general inconsistency with observed lines from the same ion. 
Perhaps the most striking features of Table 4 are the wavelength coincidences of two Y II lines and of two $\mathrm{Zr}$ II lines and also the absence of candidates for identification of three of the strongest lines. The latter three lines appear below $2600 \AA$, which means that the corresponding photon energy is about $4.8 \mathrm{eV}$. This is remarkable, considering that $4.8 \mathrm{eV}$ is not far from the highest excitation energy observed in the total spectrum and that all transitions between levels below 7-8 eV are known in ionized iron-group elements. The most probable explanation for these lines is that they originate from a neutral atom. However, as mentioned in Sect. 4.1, this region suffers from absorption which can cause a shift in the observed wavelength and a significantly decreased measured line intensity. Emission from other spatial regions can also affect the lines.

\section{Discussion}

The Sr filament proves to be a very unusual emission nebulosity. Over 600 emission lines, mostly from neutral and singly-ionized iron-peak elements, have been identified. Yet no hydrogen, helium, nitrogen or oxygen emission lines, which characterize normal emission regions, have been detected. Several factors contribute to this unlikely emission-nebula spectrum: 1) the very massive star system, while producing $5 \times 10^{6} L_{\odot}$ with a characteristic temperature of $25000 \mathrm{~K}$ (Hillier et al. 2001), has a highly clumpy, extended but cool atmosphere (Verner et al. 2003); 2) much ionized gas shields this Sr filament from hard UV radiation capable of ionizing hydrogen, and other elements requiring photo-ionizing energies exceeding $13.6 \mathrm{eV}$; 3 ) apparently the shielding may be sufficient to protect the $\mathrm{Sr}$ filament from radiation down to 6 or $7 \mathrm{eV}$ as we see no direct evidence of line emission requiring photoexcitation with that energy; 4) an intense radiation field with energies less then 6 or $7 \mathrm{eV}$ does bathe the $\mathrm{Sr}$ filament, leading to a partially-ionized region; 5) the Sr II modelling (Bautista et al. 2002) indicates a very high-density region as the electron density must be in the range of $10^{7} \mathrm{~cm}^{-3}$.

We note that the ejecta surrounding $\eta$ Car have a very nonuniform structure. While the overall Homunculus is a thin, hollow shell about ten percent thickness compared to the distance from the Central Source (Smith 2002; Smith et al. 2003b) the interior is likely a hot, low-density stellar wind. The thin surface interior to the shell is detected in [Fe II], [Ni II] emissions and Ca II absorption (Davidson et al. 2001; Smith 2002). In line of sight, ejecta absorptions of the iron-peak elements demonstrate a range of temperature and electron density that correlate with velocity (Gull et al., ApJ submitted). Interior to the Homunculus is the Little Homunculus, a miniature bilobed structure (Ishibashi et al. 2003), which is seen in multiple emission lines of iron-peak elements and in the hydrogen Balmer lines. Between the bi-lobes of the Homunculus and the Little Homunculus is the skirt region, partially seen in emission lines, and in absorption lines. Close to $\eta$ Car are several very intense emission knots, the Weigelt Blobs, seen strongly in many Fe II, Ni II, and Cr II lines (Zethson 2001). Highly-excited emission lines seen in the Weigelt Blobs and in the Little Homunculus disappeared during the 1998.0 and 2003.5 minima, but then returned. Low-excitation lines maintain constancy in flux throughout the 5.52-year period. As the Sr filament emission lines are low-excitation only, this reinforces the concept that the $\mathrm{Sr}$ filament receives radiation with the harder photons filtered out. Based upon the spatial distribution of the blue-shifted velocities, the $\mathrm{Sr}$ filament is probably located in this equatorial skirt region. At a projected distance of the order of ten light-days, the Sr filament receives intense mid-UV, and longer wavelength, radiation from the Central Source. Likely it is the strong absorption by iron and other iron-peak elements in the ionized regions and just beyond the ionized regions that shield the Sr filament. We note that the ionization potential of $\mathrm{Fe}$ is $7.9 \mathrm{eV}$ and that of $\mathrm{Sr}$ is $5.7 \mathrm{eV}$. As there is abundant $\mathrm{Fe} \mathrm{I}$ and little Fe II in the Sr filament, this indicates that the strontium is singly-ionized, but protected from becoming doublyionized by an iron-shield. Moreover, many Fe II absorptions are in the spectrum indicating the abundance of singly-ionized iron in the vicinity of the Sr filament. Shortward of $2500 \AA$, much of the ultraviolet spectrum is chopped up, further protecting the neutral and singly-ionized species with ionization potentials above 4 or 5 electron volts. Indeed the question arises as to whether molecular species might reside in this region. Groundbased, near-IR observations of the Homunculus (Smith 2002) do not indicate molecular hydrogen at these velocities or spatial position, but the ground-based observations were accomplished with lower spatial resolution.

We have systematically obtained spectra of the Sr filament to characterize the spatial extent and the level of excitation through the identification of over 600 emission lines. We have also measured the fluxes of these lines in preparation for obtaining physical information of this neutral emission region. The first paper, based upon the Sr II emission line ratios, has been published, characterizing the temperature and density of the Sr filament (Bautista et al. 2002), other papers will follow discussing models of other iron-peak neutral and singlyionized species. We hope to provide information on relative abundances of various ionic species, possibly elemental abundances, but modelling and possibly some laboratory work will first be necessary.

Acknowledgements. We are grateful to Kazunori Ishibashi for providing calibrated spectra and giving inputs in the initial analysis. Other members of the HST Eta Carinae Treasury Project Team are Manuel Bautista, Michael Corcoran, Augusto Damineli, Kris Davidson (P.I.), Fred Hamann, John Hillier, Roberta Humphreys, Jon Morse, Otmar Stahl, Nolan Walborn and Kerstin Weis. This study is part of a project funded through a contract (S.J.) with the Swedish National Space Board. The data were obtained through the following HST observational programs: 8036, 8327, 8483, 8619 and 9420. Funding was provided under the STIS GTO program and HST GO programs. We acknowledge the assistance on the analysis by the STIS Instrument Definition Team (IDT), especially Don Lindler, Terry Beck and Keith Feggans. H.H. is grateful for travel support from the STIS IDT for a visit to Goddard Space Flight Center. N.S. was supported by NASA through grant HF-01166.01A from the Space Telescope Science Institute, which is operated by the Association of Universities for Research in Astronomy, Inc., under NASA contract NAS 5-26555. This research has made use of NASA's Astrophysics Data System Bibliographic Services. 


\section{References}

Bautista, M. A., Gull, T. R., Ishibashi, K., Hartman, H., \& Davidson, K. 2002, MNRAS, 331, 875

Damineli, A. 1996, ApJ, 460, L49

Damineli, A., Stahl, O., Kaufer, A., et al. 1998, A\&AS, 133, 299

Davidson, K., \& Humphreys, R. M. 1997, ARA\&A, 35, 1

Davidson, K., Ishibashi, K., Gull, T. R., \& Humphreys, R. M. 1999, in Eta Carinae at the Millennium, ed. J. A. Morse, R. M. Humphreys, \& A. Damineli, ASP Conf. Ser., 179, 227

Davidson, K., Smith, N., Gull, T. R., Ishibashi, K., \& Hillier, D. J. 2001, AJ, 121, 1569

Gull, T., \& Ishibashi, K. 2001, in Eta Carinae and Other Mysterious Stars: The Hidden Opportunities of Emission Spectroscopy, ed. T. R. Gull, S. Johannson, \& K. Davidson (San Francisco: ASP), ASP Conf. Ser., 242, 59

Gull, T., Ishibashi, K., Davidson, K., \& Collins, N. 2001, in Eta Carinae and Other Mysterious Stars: The Hidden Opportunities of Emission Spectroscopy, ASP Conf. Ser., 242, 391

Hillier, D. J., Davidson, K., Ishibashi, K., \& Gull, T. 2001, ApJ, 553, 837

Humphreys, R. M., Davidson, K., \& Smith, N. 1999, PASP, 111, 1124
Ishibashi, K., Gull, T. R., Davidson, K., et al. 2003, AJ, 125, 3222

Johansson, S., \& Hamann, F. 1993, Phys. Scr., T47, 157

Johansson, S., \& Letokhov, V. S. 2003, Phys. Rev. Lett., 90, 11101

King, N. L., Nota, A., Walsh, J. R., et al. 2002, ApJ, 581, 285

Moore, C. E. 1945, A multiplet table of astrophysical interest (Princeton, N.J., The Observatory)

Morse, J. A., Davidson, K., Bally, J., et al. 1998, AJ, 116, 2443

Schulte-Ladbeck, R. E., Pasquali, A., Clampin, M., et al. 1999, AJ, 118,1320

Smith, N. 2002, MNRAS, 337, 1252

Smith, N., Davidson, K., Gull, T. R., Ishibashi, K., \& Hillier, D. J. 2003a, ApJ, 586, 432

Smith, N., Gehrz, R. D., Hinz, P. M., et al. 2003b, AJ, 125, 1458

Smith, N., Morse, J., \& Gull, T. R., et al. 2004, ApJ, in press

Smith, N., Morse, J. A., Davidson, K., \& Humphreys, R. M. 2000, AJ, 120,920

Verner, E., Bruhweiler, F., Verner, D., Johansson, S., \& Gull, T. 2003, ApJ, 592, L59

Verner, E. M., Gull, T. R., Bruhweiler, F., et al. 2002, ApJ, 581, 1154

Zethson, T. 2001, Ph.D. Thesis, Lund University

Zethson, T., Gull, T. R., Hartman, H., et al. 2001, AJ, 122, 322 